\title{
Enhancement of Lithium in Red Clump Stars by Neutrino Magnetic Moments
}

\author{
Kanji Mori ${ }^{1,2, *}$, Motohiko Kusakabe ${ }^{3,2}$, A. Baha Balantekin ${ }^{4,2}$, Toshitaka Kajino ${ }^{2,3,5}$, and \\ Michael A.Famiano, ${ }^{6,2}$ \\ ${ }^{1}$ Research Institute of Stellar Explosive Phenomena, Fukuoka University, 8-19-1 Nanakuma, Jonan-ku, \\ Fukuoka-shi, Fukuoka 814-0180, Japan \\ ${ }^{2}$ Division of Science, National Astronomical Observatory of Japan, 2-21-1 Osawa, Mitaka, Tokyo 181- \\ 8588, Japan \\ ${ }^{3}$ International Research Center for Big-Bang Cosmology and Element Genesis, and School of Physics, \\ Beihang University, 37 Xueyuan Road, Haidian-qu, Beijing 100083, China \\ ${ }^{4}$ Department of Physics, University of Wisconsin-Madison, Madison, Wisconsin 53706 USA \\ ${ }^{5}$ Graduate School of Science, The University of Tokyo, 7-3-1 Hongo, Bunkyo-ku, Tokyo, 113-0033 \\ Japan \\ ${ }^{6}$ Department of Physics, Western Michigan University, Kalamazoo, MI 49008 USA
}

\begin{abstract}
A recent spectroscopic survey has shown that the lithium abundances in low-mass red-clump stars are higher than theoretical prediction. Motivated by their finding, we implemented effects of additional energy losses induced by neutrino magnetic moments on stellar evolution models. It is found that the lithium abundances in red giants increase because the finite magnetic moments of neutrinos enhance the efficiency of deep mixing and ${ }^{7} \mathrm{Be}$ is conveyed from the hydrogen burning shell to the convective envelope. The results motivate further studies on the effects of non-standard energy losses on the surface compositions of low-mass red giants and red-clump stars.
\end{abstract}

\section{Introduction}

The lithium abundances in low-mass stars are long-standing problem in stellar modeling. Giant stars with $A(\mathrm{Li})=\log N(\mathrm{Li}) / N(\mathrm{H})+12>1.5$ are called lithium-rich giants [1, 2] Since stellar models predict that lithium in red giants is destroyed by the first dredge-up [3], the mechanism for the lithium enhancement in such giants is an open problem.

Lithium-rich giants with $A(\mathrm{Li})>1.5$ account for only $\sim 1 \%$ of red giants [e.g. 4]. However, a recent spectroscopic survey [5] has shown that almost all red-clump stars show the lithium abundances of $A(\mathrm{Li})=0.71 \pm 0.39$, which are higher than theoretical prediction. This observational result implies missing physical processes in stellar models.

In order to reconcile observations and models, we consider effects of additional energy losses induced by neutrino magnetic moments [NMMs; 6]. It is known that finite NMMs would make a helium core at the tip of red giant branch (RGB) heavier [7, 8]. However, their effect on the stellar surface composition has not been discussed.

\footnotetext{
*e-mail: kanji.mori@fukuoka-u.ac.jp
} 


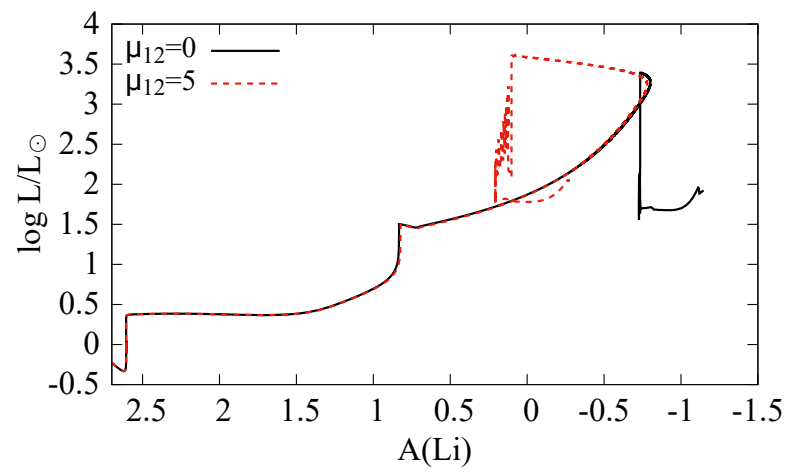

Figure 1. The luminosity $L$ and the surface lithium abundance $A(\mathrm{Li})$ of the models with $\mu_{12}=0$ and 5 .

\section{Method}

We adopt Modules for Experiments in Stellar Astrophysics [MESA; 9] to calculate stellar models. We fix the initial stellar mass to $1 M_{\odot}$ and the metallicity to $Z_{\odot}=0.0148$ [10]. We adopt the mixing length of $\alpha=1.6$. We assume thermohaline mixing as the mechanism of deep mixing. The efficiency of thermohaline mixing [11] is fixed to $\alpha_{\mathrm{thm}}=50$.

The energy loss rate induced by NMMs is given as [8]

$$
\epsilon_{\text {plas }}^{\mu}=0.318\left(\frac{\omega_{\mathrm{pl}}}{10 \mathrm{keV}}\right)^{-2}\left(\frac{\mu_{v}}{10^{-12} \mu_{\mathrm{B}}}\right)^{2} \epsilon_{\mathrm{plas}},
$$

where $\omega_{\mathrm{pl}}$ is the plasma frequency, $\mu_{\nu}$ is an NMM, $\mu_{\mathrm{B}}$ is the Bohr magneton, and $\epsilon_{\mathrm{plas}}$ is the standard plasmon decay rate.

\section{Results}

We performed simulations of stellar evolution with $\mu_{12}=\mu_{v} /\left(10^{-12} \mu_{\mathrm{B}}\right)=0-5$ [6]. Fig. 1 shows the evolution of the luminosity and $A(\mathrm{Li})$ of the models with $\mu_{12}=0$ and 5 . The star becomes more luminous as it evolves along the red giant branch. In red giants, an inert helium core is formed and its mass gradually grows because of hydrogen shell burning. When the core temperature becomes sufficiently high, helium is ignited in the core. Because the core is degenerate, helium burning at the tip of the red giant branch happens violently. As a result, the core becomes non-degenerate and stable helium burning starts. This evolutionary phase with stable helium core burning is called red clump stars.

As a star evolves in the red giant branch, the lithium abundance decreases. When $L \sim$ $0.5 L_{\odot}, A(\mathrm{Li})$ decreases by $\sim 1.5$ because of the first dredge-up, where the convective envelope penetrates into the stellar interior and lithium is destroyed by higher temperature there. The lithium abundance continues to decrease when $L>1.5 L_{\odot}$. This is because thermohaline mixing conveys the material in the envelope to the hydrogen burning shell. When the star is close to the tip of the red giant branch, $A(\mathrm{Li})$ starts increasing because ${ }^{7} \mathrm{Be}$ created in the hydrogen burning shell is conveyed to the envelope and decays to ${ }^{7} \mathrm{Li}$ [12].

When the effect of NMM is considered, the lithium abundance becomes larger around the tip of the red giant branch. This is because the additional energy loss leads to a smaller core and the density in the envelope becomes lower. The efficiency of thermohaline mixing 


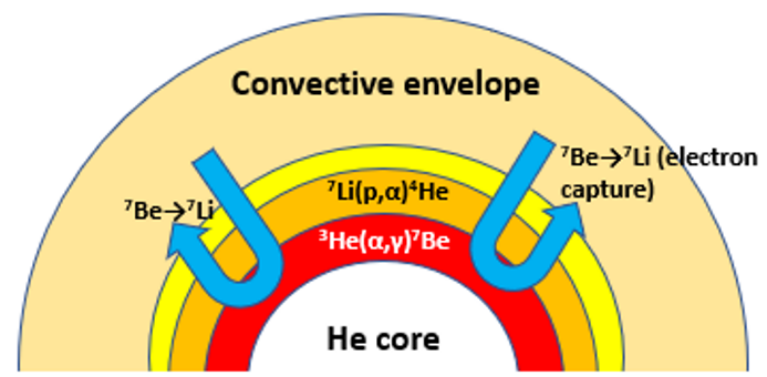

Figure 2. A schematic picture on the lithium enhancement. The figure is not to scale.

then becomes higher. As a result, ${ }^{7} \mathrm{Be}$ is conveyed to the envelope more efficiently. The mechanism of the lithium enhancement is schematically shown in Fig. 2.

Fig. 1 shows that the lithium abundance in red clump stars becomes $\sim 10$ times higher when $\mu_{12}=5$ is adopted. The enhancement of lithium may mitigate the lithium problem in red clump stars. Similar effects are expected in the cases of other physical processes such as axions. It is desirable to perform calculations with such additional energy losses induced by new physics to find a solution to the problem. Also, it is important to explore other scenarios including mixing induced by the helium flash [13] and effects of the stellar mass distribution [14].

This work is supported by Research Institute of Stellar Explosive Phenomena at Fukuoka University and JSPS KAKENHI grant number JP21K20369.

\section{References}

[1] G. Wallerstein, C. Sneden, Astrophys. J. 255, 577 (1982)

[2] J.A. Brown, C. Sneden, D.L. Lambert, J. Dutchover, Edward, Astrophys. J. Suppl. Ser. 71, 293 (1989)

[3] J. Iben, Icko, Astrophys. J. 147, 624 (1967)

[4] Deepak, B.E. Reddy, Mon. Not. R. Astron. Soc. 484, 2000 (2019), 1901.03832

[5] Y.B. Kumar, B.E. Reddy, S.W. Campbell, S. Maben, G. Zhao, Y.S. Ting, Nature Astronomy 4, 1059 (2020), 2007.07045

[6] K. Mori, M. Kusakabe, A.B. Balantekin, T. Kajino, M.A. Famiano, Mon. Not. R. Astron. Soc. 503, 2746 (2021), 2009.00293

[7] G. Raffelt, A. Weiss, Astron. Astrophys. 264, 536 (1992)

[8] M. Haft, G. Raffelt, A. Weiss, Astrophys. J. 425, 222 (1994), astro-ph/9309014

[9] B. Paxton, L. Bildsten, A. Dotter, F. Herwig, P. Lesaffre, F. Timmes, Astrophys. J. Suppl. Ser. 192, 3 (2011), 1009. 1622

[10] K. Lodders, arXiv e-prints arXiv:1912.00844 (2019), 1912.00844

[11] B. Paxton, M. Cantiello, P. Arras, L. Bildsten, E.F. Brown, A. Dotter, C. Mankovich, M.H. Montgomery, D. Stello, F.X. Timmes et al., Astrophys. J. Suppl. Ser. 208, 4 (2013), 1301.0319

[12] A.G.W. Cameron, W.A. Fowler, Astrophys. J. 164, 111 (1971)

[13] J. Schwab, Astrophys. J. Lett. 901, L18 (2020), 2009.01248

[14] J. Chanamé, M.H. Pinsonneault, C. Aguilera-Gómez, J.C. Zinn, arXiv e-prints arXiv:2109.13955 (2021), 2109.13955 